\title{
LA CONTRIBUCIÓN DE LAS EMPRESAS DE CONTADORES DE GAS ESPAÑOLAS Y EXTRANJERAS A LA ACTIVIDAD GASISTA EN ESPAÑA EN EL CONTEXTO DE LA EUROPA LATINA (1855-1936)
}

\author{
Jesús Sánchez Miñana \\ Universitat Politècnica de Catalunya \\ Email: jsminana@telefonica.net \\ ORCID iD: https://orcid.org/0000-0002-9785-8649
}

Recibido: 30 abril 2021; Aceptado 17 mayo 2021

Cómo citar este artículo/Citation: Sánchez Miñana, Jesús (2021) “La contribución de las empresas de contadores de gas españolas y extranjeras a la actividad gasista en España en el contexto de la Europa Latina (1855-1936)", Asclepio, 73 (2): p564. https://doi. org/10.3989/asclepio.2021/22

RESUMEN: Desde 1860 los fabricantes de contadores de consumo de gas o sus distribuidores debían obtener la aprobación del Gobierno para sus modelos antes de ponerlos a la venta en España. La decisión se publicaba en el periódico oficial Gaceta de Madrid, con lo que se legaba a la posteridad un valioso registro de los peticionarios. De entre ellos solo se pasa revista aquí a los fabricantes nacionales o a aquellos extranjeros que antes o después iniciaron la producción en el país, y se da cuenta de sus orígenes y vicisitudes hasta 1936, cuando el estallido de la Guerra Civil interrumpió el curso normal de las cosas. En ausencia de archivos de las empresas, el estudio ha de basarse en otras fuentes, tales como prensa y revistas, así como registros notariales y de patentes.

PALABRAS CLAVE: Contadores de Gas; Industria Auxiliar del Gas; Siglo XIX; Siglo XX; España.

\section{THE CONTRIBUTION OF SPANISH AND FOREIGN GAS-METER COMPANIES TO THE GAS BUSINESS IN SPAIN IN THE LATIN EUROPEAN CONTEXT (1855-1936)}

ABSTRACT: Since 1860 consumer gas-meter manufacturers or their distributors had to submit their models to the Government for approval before selling them in Spain. Decisions were published in the official Gaceta de Madrid, thus providing posterity with a valuable record of such entities. Among them only national producers and those foreign that sooner or later started production locally are reviewed here. An account is given of their origins and developments up to 1936 when the outbreak of the Civil War completely disrupted the normal course of events. In the absence of company archives, the study relies heavily on other sources of information such as journals and notary and patent registries.

KEY WORDS: Gas Meters; Gas-related Industry; 19th century; 20th century; Spain.

\section{NOTA PREVIA}

El contador de gas fue seguramente el primer artefacto creado para medir y poder registrar de forma continua una producción en la fábrica y su consumo por el cliente. No obstante lo intangible del producto, la pionera industria inglesa del ramo pronto pudo contar con un ingenioso aparato, el contador volumétrico hidráulico o húmedo ("wet meter") que con numerosísimas variantes y mejoras se utilizaría mayoritariamente hasta bien entrado el siglo XX, cuando comenzó a generalizarse el seco ("dry meter"), de invención antigua como el otro, pero con problemas de realización práctica que demoraron su implantación (Alayo y Barca, 2011).

Lo que sigue es el resultado de un primer estudio de la industria de los contadores de gas en España hasta la Guerra Civil de 1936, limitado a aquellas empresas que fueron autorizadas a comercializarlos y de las que se ha encontrado constancia de que llegaron a fabricarlos en el país. Así, han quedado fuera de la investigación, con dudas en algún caso, hasta quince entidades, de ellas una a finales del XIX, y las otras ya en el XX, once de las cuales posteriores a la Gran Guerra.

En este trabajo, como a menudo sucede al intentar conocer el pasado industrial, la falta de archivos de las empresas ha hecho necesario recurrir exhaustivamente a otras fuentes, incluyendo un minucioso recorrido de la prensa contemporánea, lo que multiplica el número de notas al pie. Solo se han omitido las relativas a la casa Ciervo, que pueden encontrarse en otro lugar (Sánchez Miñana y Sánchez Ruiz, 2020). 


\section{UNOS COMIENZOS CON POCOS CONTADORES}

En los inicios de la distribución canalizada de gas los primeros fabricantes no utilizaron contadores de forma generalizada para medir el consumo de sus clientes. El principal de estos, a menudo determinante de la creación de la empresa, solía ser el Ayuntamiento con el que previamente había contratado el alumbrado público, un servicio que podía perfectamente valorarse en función del número y potencia de los faroles instalados y el tiempo que habían de permanecer encendidos desde la puesta de sol. Para los abonados particulares podía seguirse de momento un criterio semejante, como hizo la Compañía Madrileña para el Alumbrado de Gas, que en 1847 estableció un cuadro de precios mensuales por tipo de mechero (de entre cuatro), "duración de la luz" desde el anochecer hasta las diez, las once o las doce, y utilización todos los días o todos salvo los domingos ${ }^{1}$. En Barcelona, sin embargo, la Sociedad Catalana para el Alumbrado por Gas estableció desde los comienzos del servicio en 1843 un sistema mixto de tarificación por tiempo para los pequeños consumidores y medición por contador para los grandes, como eran algunos establecimientos comerciales o industriales. En 1847, del total de 300 abonados particulares 107 tenían contador (Fábregas, 2020). De todos modos, entre quienes no lo tenían, los incumplimientos de contrato, bien por dar altura excesiva a la llama de los mecheros, utilizar estos con orificios de diámetro superior al pactado o prolongar el consumo más allá del horario establecido, debían de ser frecuentes, pues la Catalana en el mismo año puso avisos en la prensa local advirtiendo de las consecuencias para los infractores ${ }^{2}$.

No obstante el fraude, es posible que las empresas no se apresuraran a adoptar el contador con carácter general. Así lo creía una publicación francesa en 1861, añadiendo que "temieron ver disminuir su facturación, pretextando que los abonados tratarían de economizar todo lo posible en su consumo del gas, y que con la venta medida resultarían menos beneficios que con las eventualidades de un abono sin control" ${ }^{3}$.

\section{LOS PRIMEROS CONSTRUCTORES: RICHARDS $Y$ NALLARD}

Con más o menos lentitud, ciertamente el contador acabó generalizándose, aunque no se conocen los detalles del proceso, cuya cronología pudo ser distinta en función de las empresas, con diferencias entre las más antiguas, que hubieron de incorporar total o parcialmente el aparato a sus redes de distribución, y las nuevas que iban creándose, que quizá lo hicieran desde el principio. Señal inequívoca de la demanda creciente de unos aparatos que habían de importarse ${ }^{4}$ es la aparición de los dos primeros talleres o fábricas para construirlos en España de que se tiene noticia, el de William Richards en Barcelona y el de A. Nallard y Compañía en Madrid, respectivamente en 1855 y 1859.

Richards, ingeniero mecánico londinense, inventor de un contador seco y figura representativa de la evolución tecnológica de la industria del gas en el siglo XIX $X^{5}$, inició en 1848 una estancia de trece años en Cataluña como director de la fábrica de la Catalana. Durante ese periodo impulsó la creación de otras en el Principado, registró varias patentes relativas a los contadores hidráulicos y estableció en Barcelona un taller para construirlos que funcionó entre 1855 y 1861, año de su regreso a Inglaterra, en que pasó a su sucesora, la empresa F. Ciervo y Compañía.

Las primeras noticias encontradas de Jean-Alexandre Nallard, propietario y vecino de París, son del 5 de diciembre de 1855 , en que constituyó con Edme Courtallon, fabricante de contadores de gas en la misma ciudad, sociedad colectiva para la explotación industrial de una patente de este, relativa al perfeccionamiento de los aparatos. Con sede en la rue du Faubourg-St. Denis, 118, la sociedad se disolvió justo un año después ${ }^{6}$, pero en 1858 la fábrica continuaba funcionando en la misma dirección bajo la titularidad de Nallard y seguramente con la misma patente ${ }^{7}$, a la que él añadió otra registrada en junio siguiente ${ }^{8}$.

En 1856 el gas de Madrid había pasado a manos de la Sociedad General de Crédito Mobiliario Español, filial de la francesa Société Générale du Crédit Mobilière, e iniciado una etapa de mayor desarrollo (Arroyo, 2002). Es verosímil que, como hicieron con el carbón, los nuevos gestores procuraran asegurarse la disponibilidad local de aparatos para su industria, lo que justificaría la aparición en la capital, en 1859, de la razón social A. Nallard y Compañía, con fábrica en la hoy Ronda de Atocha, próxima a la estación de ferrocarril ${ }^{9}$. La creación de la empresa fue saludada por la prensa especializada francesa, relacionándola con la actividad del Crédito y la dirección de su "ingeniero especial" Monsieur Beau ${ }^{10}$.

Nallard, que había permanecido varios años en España, regresó a Francia en 1864 para "retomar personalmente la dirección de sus talleres y sede de París"11, que quizá no marchaban bien, pues el 1 de junio de 1866 el Tribunal du Commerce de la ciudad declaró la quiebra del negocio, siendo vendidos en subasta todos sus activos materiales unos meses después ${ }^{12}$. Por entonces aparece la que debió de ser su continuadora 
en Madrid, Sociedad Madrileña de Aparatos de Gas, cuyos anuncios de fábrica y almacenes, en los mismos lugares que su predecesora, se repiten en el semanario de economía Gaceta de los Caminos de Hierro hasta mayo de $1868^{13}$. Después, la empresa, como la de Nallard en París, quebró, pues en 1870 un juez de Madrid convocaba para el 22 de abril subasta pública de "las máquinas, útiles, aparatos, enseres y efectos que constituían la fábrica de aparatos de gas y fundición de metales de los Sres. Hubbard y compañía, sita en la Ronda de Atocha, número 15"14. El apellido en la razón social corresponde probablemente a Gustave-Nicolas Hubbard, republicano francés exiliado en España por su oposición al Segundo Imperio, fundador de la mencionada Gaceta en 1856 y su director hasta 1868 en que volvió a su país. Como se verá más adelante, la empresa Siry-Lizars situó una de las "sucursales" de su "casa central" en la dirección de Ronda de Atocha, 15, en un folleto descriptivo de los productos que llevó a la Exposición Universal de París de $1878^{15}$.

\section{NALLARD Y LA PRIMERA REGULACIÓN ESTATAL DE LOS CONTADORES}

El Real Decreto de 20 de mayo de $1855^{16}$ que aprobaba el plan de estudios de las escuelas industriales creadas cinco años antes, dispuso en su artículo 65 cuáles serían las competencias de los ingenieros que empezarían a graduarse a partir del curso siguiente. No se les reconocía ninguna en exclusiva al servicio del Estado, como era el caso de otras titulaciones, pero sí que el Gobierno se comprometía a emplearlos, "en igualdad de condiciones", en diversos cometidos, entre los que figuraba "el reconocimiento de los depósitos, tuberías y distribución del gas para el alumbrado". Después de esta declaración de intenciones no se encuentra ninguna otra referencia a la fiscalización de esas instalaciones por parte del Estado, hasta que en su número del 3 de julio de $1859^{17}$ la Gaceta de Hubbard llamó la atención sobre los contadores y la necesidad de sujetarlos a reglas que protegiesen los derechos tanto de los consumidores como de las compañías productoras, como ya habían hecho los Estados Unidos y Bélgica, y parecían próximos a hacerlo Inglaterra y Francia.

Si en el artículo ya quedaba claro que el Credit Mobilière y su filial madrileña tenían interés en que se adoptase alguna medida, otros que le siguieron en el mismo año informaron de que se había presentado al Gobierno una petición al respecto, ofrecieron detalles de las normativas inglesa, aprobada en agosto, y belga, y finalmente desvelaron que el peticionario en cuestión había sido A. Nallard y Compañía. Además en este último artículo dieron a conocer la existencia de una real orden disponiendo que la comisión de pesas y medidas, juntamente con los catedráticos del Real Instituto Industrial Eduardo Rodríguez y Magín Bonet, dictaminasen sobre el contador de Nallard y sobre las bases bajo las cuales podía plantearse en adelante la "aprobación, contraste y vigilancia" de este tipo de aparatos $^{18}$.

Resultado de todo ello fue el Real Decreto de 28 de marzo de $1860^{19}$ (Fernández-Paradas, 2016), determinando que a partir del 10 de julio siguiente los contadores para el público debían estar verificados y marcados como garantía de su aprobación y correcto funcionamiento, y "arreglados al sistema métrico". La aprobación del sistema o modelo por el Gobierno requería informe previo de una comisión, formada en Madrid por dos profesores del Real Instituto Industrial y "en provincias" dos catedráticos de la Universidad o, en su defecto del Instituto. El verificador, cargo de real nombramiento que se creaba con prioridad para los ingenieros industriales, aseguraría la sujeción de cada aparato al modelo aprobado y su correcto funcionamiento, mediante los instrumentos necesarios que le procuraría el establecimiento expendedor. Al decreto siguieron dos reales órdenes de 19 de junio, una aprobando el contador de Nallard y otra, circular a los gobernadores civiles, detallando el procedimiento a seguir por los verificadores en los laboratorios aportados por los fabricantes o vendedores, y especificando los aparatos que debían contener ${ }^{20}$. Un nueva circular del 26 siguiente a los mismos destinatarios, pospuso un mes la vigencia del decreto, al no estar "terminada la construcción de los punzones para la marca de los contadores" a que se refería en su artículo 8, y les instó a cuidar de que los establecimientos de contadores de su provincia, "si alguno existiere", solicitaran la aprobación de su sistema, tanto si era el ya autorizado de $\mathrm{Na}$ llard como otro distinto ${ }^{21}$. Conviene señalar que esta normativa de 1860 se mantuvo en vigor en sus líneas generales durante todo el periodo estudiado.

\section{CIERVO, FILIAL DE FACTO DE LA CATALANA}

F. Ciervo y Compañía fue constituida en Barcelona, el 29 de agosto de 1861, por tres socios, Salvador Capdevila Armengol, que aportaba el taller de Richards y los derechos de las patentes que le había comprado; José Galcerán Soler, que actuaba por cuenta de Claudio Gil Serra, hermano de José, hombre fuerte de la Catalana, y Federico Ciervo Pérez, que asumiría la dirección técnica. Ciervo rondaba entonces los 30 años y era en cierto modo discípulo de Richards, quien seguramente organizaría la estancia que hizo en 
Londres para formarse en la práctica como mecánico, y después le habría colocado en su negocio. Este fue, pues, continuado en el mismo local de la Barceloneta, declarando como objeto social "la construcción de contadores y otros aparatos para gas". En 1867 José Pondevida Bartrina, por cuenta de José Gil, compró sus derechos a Capdevila, con lo que la influencia de la Catalana se hizo determinante. Sin dejar de funcionar, la empresa fue reconstituida en 1872, como Federico Ciervo y Compañía, por Federico, que mantuvo sus funciones y, ya abiertamente, Claudio y José Gil. Tras la muerte de este en 1877, heredaron su participación otros miembros de la familia, y la sociedad continuó hasta que hubo de disolverse en 1885 , al alcanzar su término legal. El negocio pasó entonces a Federico a título personal.

En el transcurso de estos veinticuatro años la casa se abrió a la producción de contadores para agua, así como fogones y cocinas económicas ${ }^{22}$, y en cuanto al gas construyó aparatos para el alumbrado y amplió su oferta de equipos para las fábricas, contribuyendo además total o parcialmente al montaje de algunas y sus canalizaciones. Respecto de los contadores, no fue hasta abril de $1865^{23}$ que se aprobó el modelo que Richards había patentado en 1861 y cedido a la empresa. Esta trabajó para perfeccionar los elementos que el inventor había dispuesto en su sistema para conseguir mayor exactitud en las medidas y evitar el fraude. Las mejoras fueron objeto de dos patentes, registradas en 1871 y 1878 , e incorporadas a los fabricados, al menos las de la primera, pero no hay constancia de que se pidiera su aprobación.

Tras la compra por la Catalana de la Sociedad Anónima para el Alumbrado por Gas de Sevilla, Federico Ciervo y Cía tuvo en la ciudad una sucursal desde comienzos de la década de los 70 hasta su disolución, en que pasó a la familia Gil. Tienda céntrica con pequeño taller, ampliado después a fábrica de nueva planta en el extrarradio, sus anuncios se refieren sobre todo a lamparería.

\section{LA CASA SIRY-LIZARS EN BARCELONA}

La firma Alcide Siry, Lizars et Compagnie fue constituida en París el 18 de octubre de 1842 para dedicarse a la fabricación y venta de contadores de gas, por Michel-Alcide Siry, negociante, Charles Lizars, mecánico, socios colectivos, y un tercero de nombre no declarado en la escritura, comanditario ${ }^{24}$. En veintitrés años, a partir de 1843, registraron en Francia once patentes sobre estos aparatos, la mayor parte del sistema hidráulico y tres específicamente del seco ${ }^{25}$. Sin embargo en España solo solicitaron dos privilegios, uno en 1866, de introducción de contadores secos, seguramente correspondiente a sus últimas patentes francesas (de 1864 y 1865), y otro mucho más tarde, el 11 de febrero de 1878, de invención de un "sistema de volante medidor compensador" para los hidráulicos ${ }^{26}$. Incorporaba esta novedad uno de los dos contadores que la casa llevó a la Exposición Universal de París de ese año, presentado como "de medida invariable" en un folleto ya citado $^{27}$, y que parece fue también el que sometió a aprobación en España, concedida por Real Orden de 13 de enero de $1879^{28}$.

En el folleto figura la sucursal de Madrid, como queda dicho, y también la de Barcelona, en la calle del Carmen, 30. Es posible que ambas vinieran gestionando la importación de contadores y manteniendo un taller de reparación, pero no fábrica. En Madrid Siry- $L i$ zars habría empezado a venderlos a la compañía del gas, después de la quiebra de la sucesora de Nallard, y en Barcelona la empresa de Charles Lebon, que inició el suministro en 1865-1866, compitiendo para los particulares con la Catalana, podría también ser cliente habitual de la empresa francesa ${ }^{29}$. Sea como fuere, sí parece claro que asegurando privilegio y aprobación para su contador, Siry-Lizars iniciaba un plan para aumentar su presencia en España.

Efectivamente, en el mismo 1878 hay noticias de dos presentaciones que hizo en Barcelona, en su taller de la Ronda de la Universidad, no 96, a las que invitó a la prensa. El director de la sucursal, que había suscrito la petición de aprobación del contador, se llamaba Eugenio Anglés y se presentaba como "caballero de la Legión de Honor"30. Seguramente era el Eugène-Joseph-Antoine-Bernard Anglès, poseedor de esa distinción y capitán retirado de la infantería de marina francesa, autorizado por su Gobierno en aquel año a residir temporalmente en Barcelona ${ }^{31}$. Las demostraciones tuvieron lugar el 18 de julio ${ }^{32}$ y el 30 de octubre ${ }^{33}$, la primera con asistencia también, entre otros, del director de la Escuela de Ingenieros Industriales, Ramón de Manjarrés y de Bofarull y el ingeniero verificador de la provincia Magín Lladós y Rius $^{34}$. Se conoce la reacción de este, que habría instado al Gobierno nada menos que a imponer el uso del contador Siry-Lizars en sustitución de los existentes ${ }^{35}$, y el informe favorable de otros dos ingenieros industriales, José María Rodríguez Carballo y Francisco de Paula Rojas y Caballero-Infante ${ }^{36}$.

La casa Ciervo, que había asistido a la presentación de julio, no reaccionó públicamente hasta que en el periódico de Barcelona La Imprenta apareció, el 29 de enero de $1879^{37}$, un elogioso artículo sobre el contador 
de su competidor, tras del cual es verosímil que este se encontrara, al menos como inspirador. Al aparato de Siry-Lizars "le falta poco para ser perfecto -escribierony desde luego cabe asegurar que de todos los sistemas es el más exacto y el más fiel que hasta hoy ha podido construirse". Federico Ciervo y Cía. respondió en la prensa con un "remitido", Siry-Lizars, por boca de Anglés, le contestó con otro, y ambos volvieron a cruzarse escritos dos veces más ${ }^{38}$. Y a la pequeña guerra de remitidos unieron la publicidad. Primero fueron anuncios breves, que inició Ciervo el 8 de febrero en el Diario, seguido el 13 por Siry-Lizars que insertó los suyos en la misma sección, pero al año siguiente los textos fueron más largos y agresivos. Sirva de muestra uno de Siry-Lizars, que incluía la frase "Garantía de exactitud: el sello del Gobierno”, seguida de esta advertencia: “iPúblico! Para evitarte muchos perjuicios, rechaza, según tu derecho, todo contador que no lleve la garantizada MEDIDA INVARIABLE, por más que algunos interesados abogan por el contador de nuestro competidor que permite el FRAUDE, aunque esté SELLADO por el delegado del Gobierno" ${ }^{39}$. O este de Ciervo en el que se leía: "La confianza que se nos dispensa y el carácter propio de constructores hijos del país nos eximen de insertar pomposos anuncios que no tienen más objeto que sorprender al público con charlatanerías" ${ }^{\prime 0}$.

En 1881 la casa Siry-Lizars, junto con otras de Francia, Bélgica y Holanda, se integró en la Compagnie pour la Fabrication des Compteurs et du Matériel pour les Usines à $G a z$ (en adelante CFC), al crearse en París por transformación en sociedad anónima de la comanditaria del mismo nombre, constituida en 1877, cuya razón social era M. Nicolas, G. Chamon, Foiret et Compagnie (Pelpel, 1987) ${ }^{41}$. Ya en 1882, entre los donantes a una causa benéfica figuraba en un periódico de Barcelona la "Compañía para la fabricación de contadores para gas, en la sucursal de la calle de Balmes, núm. 6", seguida de "Eugenio Anglés, director" y una relación de trabajadores. En la lista hay un contramaestre, ocho operarios y un aprendiz, suficientes quizá para dotar un pequeño taller de fabricación, haciendo honor al nuevo nombre de la empresa ${ }^{42}$. Al año siguiente Anglés anunciaba en la misma dirección "Contadores para gas hierro colado" ${ }^{43}$.

\section{MOVIMIENTOS EN LA SUCURSAL DE CFC Y NUEVA FÁBRICA DE CIERVO}

En 1887 los contadores Siry-Lizars de la sucursal barcelonesa de CFC ya no salían de Balmes, 6 , sino de "los grandes talleres situados Ronda de San Antonio, 52 y $54^{\prime 44}$. Aquí seguían funcionando en 1889 y $1890^{45}$, pero los anuncios los sitúan entre 1892 y 1897 en la calle del Olmo, $10^{46}$, y en 1900 comienzan a documentarse en Santa Madrona, $15^{47}$. Una breve noticia del 17 de diciembre de 1901, relativa a la huelga de metalúrgicos que reclamaban la jornada de nueve horas, permite conocer el tamaño de la plantilla de entonces, muy crecida desde la época de Balmes: "De los ochenta obreros empleados en la fábrica de contadores que el señor Anglés tiene establecida en la calle de Santa Madrona, trabajaron ayer treinta"48. Este incremento seguramente explica los repetidos cambios de ubicación de la empresa, cada vez necesitada de más espacio, consecuencia de una mayor demanda y una oferta muy diversificada, que incluía otros artículos para el gas y contadores para agua al menos desde $1889^{49}$, y contadores para electricidad desde el año siguiente ${ }^{50}$.

Pudieron ser anteriores, pero no es hasta 1902 que se constatan también cambios al frente de la sucursal, quizá motivados por la edad u otras circunstancias de Eugenio Anglés, que llevaba un cuarto de siglo a su cargo. Los directores eran entonces dos: él mismo y José Anglés, quizá su hijo ${ }^{51}$. Pero esta situación continuaría por poco tiempo, pues en 1905 había de nuevo un director único, el colombiano Andrés Elías Lorenzo Triana Umaña, titulado ingeniero por la École des Ponts et Chaussées de París en 1883, con un historial profesional en el campo de los ferrocarriles y la minería en diversos países ${ }^{52}$.

En el periodo de veinte años en que Federico Ciervo García fue único propietario y director, su Casa debió de conocer también un periodo de expansión que llevó a la construcción de una fábrica de nueva planta sobre una parcela de más de $3.200 \mathrm{~m}^{2}$, en la calle de Ginebra de la Barceloneta, inmediata a la anterior ubicación de la calle del Norte. El seguimiento que hizo un periódico de la huelga de metalúrgicos de 1901-1902, proporciona también datos sobre el número de sus trabajadores: el 24 de febrero de 1902 se presentaron "de treinta a cuarenta operarios, habiendo dejado de hacerlo $41^{\prime \prime 53}$. La empresa tenía, pues, por entonces un tamaño parecido al de CFC, compensando seguramente su ausencia del ramo de los contadores eléctricos, en el que nunca llegó a entrar, con una mayor presencia en el del agua, ampliado a las bombas elevadoras, y el de las cocinas económicas. Todo ello sin contar su actividad en el diseño y montaje de plantas de gas, protagonizada especialmente por el hijo mayor del dueño, José Ciervo Sinclair, ingeniero industrial desde 1881.

En lo que podría indicar un cambio en la relación con su competidor, Federico Ciervo, poco antes de su muerte, ocurrida el 1 de octubre de 1905, suscribió conjuntamente con Andrés Triana dos solicitudes re- 
lativas a las "instrucciones reglamentarias para el servicio de verificación de los contadores de electricidad y de gas", aprobadas por Real Decreto de 7 de octubre de $1904^{54}$. En el caso del gas, la norma establecía una nueva regulación estatal, en sustitución de la menos extensa y detallada contenida en las anteriores de 1860 y $1880^{55}$. Seguramente intentaba satisfacer las demandas de los abonados, que en los últimos años habían sido particularmente críticos con las empresas suministradoras (hasta el punto de que diversos gremios declararon una huelga de consumo que se desarrolló en Barcelona en marzo-abril de 1901), y venían señalando a los contadores como un medio del que las gasistas se valían para defraudarles ${ }^{56}$.

En el primer escrito, de 18 de mayo de 1905, Ciervo y Triana solicitaron la modificación de hasta doce artículos de las "instrucciones", a algunos de los cuales ya habían objetado sin éxito la empresa del gas de Madrid y las dos de Barcelona, así como otras que se les adhirieron. La solicitud fue remitida a los verificadores de esas dos ciudades, que en su informe recomendaron dejar las "instrucciones" como estaban y aprovecharon para pedir otras cosas de su cosecha, la más destacada la obligatoriedad de que los contadores llevaran la llamada "caja de agua" para evitar explosiones por salida del gas al manipularlos. Todo lo hizo suyo el Ministerio en su respuesta por Real Orden del 3 de agosto ${ }^{57}$, y también denegó el 22 de septiembre la segunda petición que habían hecho Ciervo y Triana, seguramente el mes anterior, de que se ampliara el plazo de dos años en que debía incorporarse dicha "caja" a todos los contadores ya instalados o por instalar ${ }^{58}$.

Triana sí consiguió que se aceptara su petición de 26 de octubre de 1906 para que volvieran a verificarse los contadores "Duplex" de CFC que, no obstante haber sido aprobados cuatro años antes a instancia de José Anglés, el correspondiente servicio de Barcelona se negaba últimamente a admitir, alegando que no reunían los requisitos exigidos por el Decreto de 8 de junio de 1906, que había modificado las "instrucciones" del de $1904^{59}$. También en 1906 le fue autorizado un aparato de previo pago aplicable "a los contadores de gas de sistemas aprobados" 60 .

\section{LOS CONTADORES BOIX}

El probable duopolio que constituían los fabricantes Ciervo y CFC terminó a principios del siglo XX con la aparición de un nuevo contador hidráulico autóctono, denominado "el invencible" por sus inventores, Pedro y Juan Boix Alafalla, que el 30 de septiembre de 1901 obtuvieron la autorización para su uso ${ }^{61}$. Antes, el 4 de julio, Pedro, junto con Ramon Planell Pedrol, había pedido la correspondiente patente, que les fue concedida el 3 de agosto ${ }^{62}$. Nada se ha podido averiguar sobre Planell, y de los Boix solo se sabe que eran barceloneses, hijos de un tejedor, y que en 1901 Juan cumplía 44 años, ignorándose la edad de Pedro ${ }^{63}$. Cabe pensar que el gran conocimiento de los contadores que al menos uno de ellos -seguramente Pedro-debía de tener, fuera fruto de su experiencia en un taller donde se construyeran o repararan.

El 17 de noviembre del mismo año, Pedro, "industrial"; Juan, "del comercio", y Cicio Boix, socios colectivos, y los hombres de negocios Domingo Taberner Prims y Joaquín María Tintoré Mercader, comanditarios, todos vecinos de Barcelona, salvo Juan que lo era de Terrassa, constituyeron en aquella ciudad, por cinco años, Boix Hermanos, S. en C., para explotar el "invencible". Llevaría la gerencia y firma social Cicio, entonces capitán de Infantería ${ }^{64}$, Pedro aportaba la patente, de la que Planell le había cedido su parte, y Taberner y Tintoré el capital, 50.000 pesetas, a partes iguales ${ }^{65}$. La fábrica o taller se instaló en la calle de Sant Pau, 93, donde la sitúa una revista a finales de 1902, que da detalles constructivos del contador "invencible" y también de otro, denominado "el económico"66, más sencillo y barato, cuya patente Pedro Boix acababa de solicitar ${ }^{67}$ y sería aprobado por Real Orden de 9 de enero de $1903^{68}$.

La ya mencionada Real Orden de 3 de agosto de 1905, denegando a Ciervo y Triana su petición de modificación de diversos artículos del decreto que había reglamentado la verificación de los contadores, remite al informe de los verificadores de Barcelona. Según estos, la solicitud de los dos industriales "tiende únicamente a procurar su personal provecho", y la prueba es que Boix Hermanos no ha firmado con ellos y lo ha justificado en un comunicado, publicado en el Diario de Barcelona del 30 de mayo de $1905^{69}$, porque sus aparatos cumplen las disposiciones del decreto y "sobre garantir debidamente los intereses de los consumidores de fluido, colocan la industria española de fabricación de contadores en el lugar que la corresponde, y en consecuencia con los adelantos modernos".

La opinión de los verificadores, que la empresa hubo de conocer desde que salió al mercado, pudo animarle a que pidiera la convocatoria de un concurso nacional para que fuera elegido y declarado obligatorio un determinado sistema de contadores. No tuvo éxito ${ }^{70}$, pero aprovechando la propaganda gratuita que le había hecho la Gaceta de Madrid, reprodujo la real orden completa en un anuncio, al que añadió un pie explicando que con ello pretendía dejar claro que sus 
contadores eran "los más perfeccionados, con arreglo a las disposiciones vigentes, y marcando igual a todas las presiones", "en vista de las grandes luchas" que había tenido que soportar "durante los cuatro años de su fundación, a causa del menosprecio que daban a sus aparatos personas poco escrupulosas"71. Pero las luchas pronto acabaron resultando infructuosas y la sociedad no llegó a cumplir los cinco años previstos, ya que según los estatutos debía disolverse "por pérdida de un cincuenta por ciento del capital", lo que, dándose esta circunstancia "con exceso", se acordó el 17 de marzo de $1906^{72}$. La subasta judicial de todo su activo, incluida la patente del "económico" (la del "invencible" había caducado), se anunció para el 20 de diciembre de $1907^{73}$ en el taller de Sant Pau, 93. Es posible que el adjudicatario fuera $L$. de la Torre, que en 1911 fabricaba en esa dirección aquellos contadores ${ }^{74}$.

Las noticias de 1912-1913 de una nueva huelga de metalúrgicos en demanda de la jornada de nueve horas, descubren la existencia de una tercera empresa de contadores de gas afectada, además de las de Ciervo y Triana. Se llamaba Hijos de J. Abellanet, sucesora de una acreditada fundición de latón y cobre en Manresa, con talleres aquí y en Barcelona, este precisamente en la misma ubicación de $L$. de la Torre, y por tanto aparente continuador suyo. Su cabeza visible era Casimiro Abellanet y Fabra, dirigente local del partido liberal histórico ${ }^{75} \mathrm{y}$, por cierto, último en satisfacer las demandas de sus 42 obreros tras 235 días de paro (Sastre, 1915) ${ }^{76}$. El 2 de marzo y 14 de abril de 1914, respectivamente, Pedro Boix le cedió sendas patentes nuevas de 1910 y 1911, correspondientes a un contador que llamó "el constante" y a un aparato de pago previo para adicionarle ("el torpedo") ${ }^{77}$, que le fueron autorizados por reales órdenes de los mismos años ${ }^{78}$. En estas figura domiciliado en Manresa, cambio de residencia que sugiere, como las cesiones, una posible relación laboral o societaria con Abellanet, en el negocio de los contadores. Este, iniciado en Hijos de J. Abellanet, debió de continuar bajo la razón social $\mathrm{Ca}$ simiro Abellanet, S. en C., que figura en la placa de un contador de 1915, "sistema Boix", puesto a la venta en Internet $^{79}$. La inscripción de la empresa en el Registro Mercantil, si se hizo, no se ha encontrado, y solo se ha podido conocer la dirección de la fábrica ${ }^{80}$. Parece probable que su existencia no se prolongara más allá del fallecimiento de su titular, en $1933^{81}$.

\section{LOS SUCESORES DE FEDERICO CIERVO GARCÍA}

En 1906, unos meses después de la muerte su padre, José y Federico Ciervo Sinclair, constituyeron Hijos de Federico Ciervo, S. en C., para "la fabricación y venta de artículos para toda clase de alumbrado, calefacción e hidráulica, y especialmente de contadores y material para fábricas de gas". Ciertamente toda esa actividad debió de continuar, en el caso de los contadores con un nuevo modelo hidráulico, adaptado a la normativa vigente, patentado inmediatamente y autorizado en los primeros días de 1907 . No obstante, la creciente competencia y el derecho concedido a los abonados a utilizar contadores de su propiedad de cualquier sistema aprobado ${ }^{82}$, llevarían a la empresa a buscar otros campos de negocio, como el de las instalaciones eléctricas, con una breve incursión en la fabricación de conductos aislantes. Lo mismo sucedió con su sucesora Hijo y Nietos de Federico Ciervo, S. L., establecida por José con sus dos hijos en 1926 a la muerte de Federico, que en 1934 producía también material contra incendios.

\section{CFC CONVIERTE LA SUCURSAL ESPAÑOLA EN SOCIEDAD FILIAL}

Por acuerdo del consejo de administración de CFC el 24 de diciembre de 1907, su sucursal de Santa Madrona, 15, pasó a ser Chamon y Triana, Sociedad en Comandita. La constituyeron, siete días después, por cinco años prorrogables otros cinco, Triana en nombre propio y por representantes CFC y Gabriel Chamon Bowers, el ya citado "G. Chamon" (su madre se apellidaba Bowers) de la razón social de la empresa comanditaria que en 1881 se transformó en CFC (Giguel, 2014). Chamon y Triana, socios industriales, tendrían la gerencia y firma, y CFC sería el socio comanditario, aportando el activo de la sucursal, valorado provisionalmente en 750.000 pesetas, y unos terrenos recientemente adquiridos de la Granja Avícola de San Luis, antes "Can Poc Oli", en Sarrià. Este capital fue incrementado en 50.000 pesetas en efectivo por Chamon, que pasaba así a ser socio comanditario, el 17 de febrero siguiente ${ }^{83}$. De 1908 son también la solicitud por la nueva entidad de una patente de introducción y los primeros registros de una marca comercial de contadores de agua ${ }^{84}$.

Según algunas reseñas publicadas sobre la participación de CFC en la Exposición Internacional de Higiene, Artes, Oficios y Manufacturas, celebrada en Madrid en 1907, ya entonces la empresa habría iniciado la construcción de una nueva fábrica ${ }^{85}$. Levantada en la carretera (hoy avenida) de Sarrià, número 48, y de notable arquitectura por las bóvedas tabicadas de las naves ${ }^{86}$, el traslado se celebró el 29 de enero de 1911 con un banquete a la muy crecida plantilla de la empresa, 250 personas, en cuyo transcurso fueron homenajeados cuatro obreros veteranos, uno de ellos 
con treinta y cuatro años de servicios, o sea desde los inicios como Siry-Lizars ${ }^{87}$. La visita a las instalaciones en abril de 1913 por los participantes en el Primer Congreso Nacional de Industrias Metalúrgicas fue objeto de reseñas en varias publicaciones, según las cuales la planta, todavía no del todo terminada, ocupaba una superficie de $10.000 \mathrm{~m}^{2}$, empleaba a 300 personas y producía mensualmente unos 3.000 contadores de gas, más de 1.000 de electricidad y otros tantos de agua. Las informaciones destacan las condiciones laborales: trabajo mayoritariamente a destajo en un espacio desahogado, con luz y temperatura adecuadas, primas a la producción, media jornada los sábados y médico y medicinas gratis para los empleados ${ }^{88}$.

Durante su gerencia, Triana, además de conseguir en 1906 la aprobación del contador de gas "Duplex", obtuvo la de otros tres de tipo hidráulico, uno en 1912 y dos en $1917^{89}$, todos dotados del llamado "compensador Dupoy", un aparato sin órganos mecánicos, inventado por el ingeniero francés Jean Romain Dupoy para mantener constante el nivel del agua ${ }^{90}$. Los de 1917 fueron sendos "Duplex" de la Casa para tres y cinco mecheros, pero provistos del compensador y un mecanismo de pago previo ${ }^{91}$.

La sociedad, expirado su plazo legal tras la prórroga por un segundo periodo de cinco años, fue definitivamente disuelta el 29 de abril de 1918 por CFC, que tenía la potestad estatutaria para hacerlo, no sin incluir en la escritura el activo del balance general a 31 de marzo, con una importante disminución del capital social que ya no llegaba a 440.000 pesetas $^{92}$. La empresa siguió funcionando con la constitución, el 30 de abril, de G. Chamon, Sociedad en Comandita, por CFC y Chamon, que aportó el capital de 100.000 pesetas. El gerente era Chamon, que encontrándose ausente y "dadas las dificultades en comunicaciones por el estado actual europeo", delegaba la gestión y poderes en dos residentes, Paul Viteau, ingeniero, y Carlos Camacho, “del comercio"93. La nueva razón social participó en 1920 en la primera Feria Oficial de Muestras de Barcelona, presentando, además de los contadores de agua, gas y electricidad, otro equipamiento eléctrico, como aparatos de medida y aisladores ${ }^{94}$.

Al año siguiente, el 22 de abril, fue disuelta por Chamon en persona y CFC, y acto seguido la antigua sucursal fue convertida en la sociedad anónima de duración indefinida, Compañía para la Fabricación de Contadores y Material Industrial, y se protocolizaron sus estatutos. El capital se estableció en 1.800 .000 pesetas, en acciones de 500, suscritas en su totalidad por los cuatro constituyentes: Chamon, 3.000, y Joseph-Ma-
rie-Louis Urtin, abogado de París; Alejandro Jaumandreu Puig, abogado de Barcelona, y Salvador Navarro Sanjuán, "agente de transportes" de la misma ciudad, 200 cada uno. El primer consejo de administración, celebrado a continuación, integrado por Chamon, presidente, Jaumandreu, vocal, y Navarro, secretario, acordó delegar en Viteau y Camacho la gestión de la sociedad ${ }^{95}$, que en el mismo año emitió obligaciones por valor de 900.000 pesetas $^{96}$. Entre 1926 y 1931 la sucursal en Madrid, calle Juan de Mena, 5, anunciaba, además de los productos presentados en la Feria de 1920, "instalaciones completas para distribución de gasolina" y "talleres para la reparación de toda clase de contadores"

En 1925 Camacho obtuvo para la CFC española la aprobación del contador "Sigma", que la casa madre ya había patentado aquí como "contador de gas de campanas oscilantes" cinco años antes. Era un aparato hidráulico (utilizaba aceite de vaselina en lugar de agua), pero basado en el principio de funcionamiento de los secos, y en 1929 se autorizó dotarle del mecanismo de pago previo aprobado en $1906^{98}$.

\section{LOS CONTADORES SECOS DE KROMSCHROEDER Y ELSTER}

Los hermanos Heinrich Hermann y Johann Friedrich Gustav Kromschroeder, de Osnabrück (Baja Sajonia, Alemania), que trabajaban con Samuel Clegg, inventor del contador hidráulico, en Inglaterra, registraron aquí tres patentes de contadores de gas. Fueron dos relativas a los hidráulicos en 1862, una solo de Heinrich y la otra conjunta, como también lo fue la de 1865 sobre un contador seco ${ }^{99}$. Las aportaron a la recién constituida The London Gas-Meter Company, Limited, que puso una sucursal en Osnabrück bajo la dirección de otro de los hermanos, Georg. Este, al desaparecer muy tempranamente la matriz por dificultades de financiación, continuó por su cuenta, estableciendo en su ciudad la G. Kromschröder Fabrik für Gasuhren, que en 1874 pasó a ser G. Kromschroeder, Aktien Gessellschaft ${ }^{100}$.

Un sucesor evolucionado del contador seco de 1865 fue el primero de este tipo aprobado en España, por Real Orden de 7 de agosto de $1908^{101}$. Para él se autorizó también un aparato de previo pago el 11 de marzo de 1910, y una simplificación de este el 27 de agosto de $1912^{102}$. Pasada la Gran Guerra, en 1922 fue aceptado el sistema llamado "III a", y después sus versiones "1925", "1930" y "1932", así como dispositivos de pago previo para los dos últimos ${ }^{103}$. 
La sucursal española se estableció probablemente en Barcelona, donde a partir de 1922 hay constancia de su dirección, calle Balmes, 47, en que se anuncia como fábrica de contadores ${ }^{104}$. El 20 de febrero de 1932 se convirtió en filial como Sociedad Anónima Kromschroeder, de duración indefinida, que constituyeron Faustino Ballvé Pallisé, abogado, en nombre propio, y Friedrich Kromschroeder por la matriz de Osnabrück, suscribiendo entre ambos la totalidad de las 300 acciones de 1.000 pesetas, representativas del capital, 100 el primero y 200 el segundo. Cuatro días más tarde fueron apoderados para llevar conjuntamente la empresa Kurt Kromschroeder y Artur Schlinder ${ }^{105}$, este ya vinculado a ella, dado que había suscrito como su representante todas las solicitudes de aprobación de contadores a partir de la concedida en 1927. El 2 de marzo de 1935 se acordó duplicar el capital social ${ }^{106}$ y en 1936 la fábrica estaba en la calle Industria, $278^{107}$.

Después de Kromschroeder, la empresa Elster \& Co. Gasmesserfabrik, A. G., de Maguncia, fue en 1912 la segunda en obtener la autorización para comercializar un contador de gas seco, el "Emil Haas"108, por el nombre de su inventor y partícipe de la sociedad, que patentó su primera versión en $1878^{109}$. En 19311933 se aprobaron los modelos $\mathrm{HO}$ y $\mathrm{HL}$, y un dispositivo de pago previo para ellos ${ }^{110}$. Por entonces debió de constituirse Elster, S. A., probablemente en 1933, año en que el presidente de su consejo de administración, Antonio Gabriel Rodríguez, obtuvo el permiso para que el nombre de la filial sustituyera en los contadores aprobados al de la matriz, pues esta le había "cedido todos sus derechos de fabricación y venta en España"111. La Sociedad, domiciliada en la calle Luis Mitjans, no 4, de Madrid ${ }^{112}$, no se ha localizado en el Registro Mercantil de la ciudad.

\section{NOTAS AL FINAL}

1 Gaceta de Madrid (en adelante Gaceta), 11-II-1847, p. 1. Diario de Barcelona, 26-II-1847, p. 932, y 23-XI-1847, p. 5557. Portefeuille économique des machines, de l'utillage et du matériel, Janvier 1861, pp. 4-11, en “Étude général sur les compteurs à gaz" por M. G. Jouanne.

4 Sirva de ilustración la noticia de la arribada al puerto de Barcelona, el 11 de mayo de 1854, de un bergantín procedente de Leith (Edimburgo) "con 135 toneladas carbón piedra y 36 cajas contadores para gas a D. José Gil [Serra]", al frente entonces de la Catalana, en Diario de Barcelona del día siguiente, p. 3424.

5 The Gas World, 20-V-1893, p. 536, escribió en su necrología: "His removal breaks another of the few remaining links which connect the gas industry of the past with that of the present".

\section{REFLEXIÓN FINAL}

Aunque se haya repetido tantas veces con distintos protagonistas y tecnologías, no deja de llamar la atención el caso Ciervo versus Siry-Lizars, pioneros ambos en sus respectivos países. Ya en 1881, en un elogioso artículo para la casa barcelonesa, una revista de Madrid le animaba a expandirse, introduciendo sus contadores en la capital, donde probablemente imperaban los de Siry-Lizars, pero también le recomendaba "despejarle el porvenir a sus talleres", abordando el ramo de los contadores para electricidad ${ }^{113}$. Seguramente Ciervo estuvo de acuerdo, entró en el campo de los contadores para agua, pero hacerlo en el de los eléctricos hubiera supuesto, entre otras cosas, modificar su condición de negocio familiar y sobre todo un gran incremento de recursos de capital, que su competidor, finalmente convertida en la muy poderosa CFC, sí acometió. A esta empresa, además, diversificar su producción debió de permitirle compensar, mucho más que a Ciervo que también lo intentó, el muy probable descenso de ventas de sus contadores hidráulicos por la irrupción de los secos que la industria alemana venía desarrollando desde hacía años. En medio de este panorama, una pequeña fábrica, como seguramente fue la de Boix, después $A b e$ llanet, hubo de tener poco porvenir aunque su producto, en cierto modo anticuado por hidráulico, fuera muy bueno en opinión de los expertos.

\section{AGRADECIMIENTOS}

Este artículo forma parte de los resultados del Proyecto I+D+I de Excelencia, PID2020-112844GB-100, "El gas en la Europa Latina: una perspectiva comparativa y global (1818-1945)", financiado por el Ministerio de Ciencia e Innovación del Gobierno de España y Fondos Feder.

6 Gazette des tribunaux, París, 9-XII-1855, p. 1172, y 29-XI1856, p. 1166.

7 En anuncio de primera página de la revista Le gaz, París, 30IV-1858, la fábrica de "Adre Nallard" ofrece, entre otros artículos, "Compteurs à gaz / Système breveté s. g. d. g. [sens garantie du gouvernement]".

8 Courtallon, patente $\mathrm{n} 022.800$, "perfectionnments apportés aux compteurs à gaz", depositada el 12-III-1855. Nallard, patente no 36.851 , "compteur à gaz à niveau constant", depositada el 1-VI-1858, con una adición el 28-V-1859. Institut National de la Propriété Industrielle (INPI), París.

9 Anuncio en Gaceta de los Caminos de Hierro, 3-VII-1859, p. 413.

10 "Le gaz en Espagne", Le Gaz, 20-III-1859, p. 40.

11 Le gaz, 30-IV-1864, p. 45.

12 Véanse los periódicos de París Le Temps, 3-VI-1866, p. 3; Le Pays, 21-VII-1866, p. 3, y La Liberté, 5-VIII-1866, p. 4. 
13 El primero figura en el número de 8-IX-1866, p. 573, y el último en el de 17-V-1868, p. 325.

14 Diario Oficial de Avisos de Madrid, 13-IV-1870, p. 2.

15 "Exposition Universelle de 1878 / Note sur les appareils exposés par A. ${ }^{\text {de }}$ Siry, Lizars \& $C^{\text {ie }}[$ [...] Paris / Alcan-Lévy Imprimeur [...] 1878".

16 Gaceta del 22 y 23 , pp. $1-2$ y 1.

17 P. 410

18 Gaceta de los Caminos de Hierro, 23-X, 6-XI, 13-XI y 18-XII1859 , pp. 662, 695-696, 710-711 y 791

19 Gaceta del 30, p. 1

20 Gaceta, 24-VI-1860, pp. 1-2. Nallard no solicitó ningún privilegio en España para su contador de gas; sí uno de introducción de un contador para agua basado en el sistema de turbina de Siemens. Archivo Histórico de la Oficina Española de Patentes y Marcas (AHOEPM), exp. PR 2299.

21 Gaceta, 27-VI-1860, p. 1.

22 Anuncios ilustrados de los dos últimos en El Porvenir de la Industria, Barcelona, 8-IV-1875, y algunos números siguientes de ese año.

23 Gaceta, 27-IV-1865, p. 1

24 Reseña de la escritura en Le Droit, Paris, 31-X-1842, p.4. El nombre de Siry se encuentra después abreviado en las men-

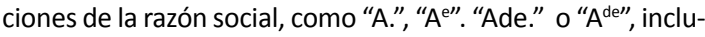
so erróneamente como "A. de".

25 INPI.

26 AHOEPM, exps. 4122 y 5792.

27 Descripción más completa que la del folleto, en El Porvenir de la Industria, 17-V-1878, p. 166 y lámina 24.

28 Gaceta, 21-I-1879, p.195. A diferencia de las órdenes anteriores relativas a los contadores de Mallard y de Ciervo, esta no incluye una descripción del aparato, y solo dice que es "de medida invariable y sin peligro de explosión".

29 La Corona, Barcelona, 30-VII-1867, edición de la tarde, p. 3, copió de Le Gaz, París, 31-V-1867, p. 60, la noticia de la construcción por Siry-Lizars en Francia para la fábrica de gas Lebon de Barcelona, de un contador de gran tamaño, del modelo utilizado en el alumbrado del Champ-de-Mars para la Exposición Universal de París de aquel año. Nótese que dos días después, en su edición de la mañana, p. 3, el periódico escribía que les habían dicho que un contador de la Catalana era aun de mayores dimensiones, "producto del establecimiento de los señores Ciervo y compañía sito en la Barceloneta, y que en nada cede por la perfección de sus artefactos a las fábricas más reputadas del extranjero".

30 La Imprenta, 23-I-1879, p. 499.

31 "Bulletin des lois de la République Française [...] deuxième semestre de 1878 [...] partie supplementaire. Tome XVII [...] MDCCCLXXVIII", p. 14

32 Diario de Barcelona, 19-VII-1878, ed. de la mañana, 8370, y Boletín mensual de la Asociación de Ingenieros Industriales de Barcelona, no 6, IX-1878, p. 11.

33 La Publicidad, Barcelona, 30-X-1878, p. 2.

34 La había anticipado en el artículo citado El Porvenir de la Industria, revista cuyo creador y director era Lladós, verificador desde 1860. "Según tenemos presentido-escribieron-la sucursal de dicha casa constructora establecida en esta capital, calle de Ataulfo número 44 [¿̇dirección posterior a la de calle del Carmen y anterior a la de Ronda de la Universidad?], va a presentar algunos de estos aparatos".

35 A la iniciativa de Lladós se refiere con elogios, dando su cargo pero no su nombre, la "exposición" o preámbulo del Real Decreto de 13 de febrero de 1880 (Gaceta del 14, p. 407).
La disposición, primera sobre verificadores y sus atribuciones después de la instrucción de 19 de junio de 1860, no recoge su propuesta sobre el contador de Siry-Lizars, pero sí otras medidas al parecer contenidas en la memoria que había dirigido al Gobierno.

36 Boletín mensual de la Asociación de Ingenieros Industriales de Barcelona, no 9, XII-1878, pp. 9-10.

37 Ed. de la mañana, pp. 674-675, en "Crónica local".

38 Pueden verse sucesivamente en Diario de Barcelona, 2 y 9-II1879, pp. $1418-1419$ y $1703-1705$, y en El Diluvio, Barcelona, ed. de la mañana, 16, 23, 25-II y 1-III-1879, pp. 178-180, 360361, 396 y $495-496$.

39 La Publicidad, 1-II-1880, p. 4 (mayúsculas del original).

40 El Diluvio, 14-III-1880, p. 2026 (negritas del original).

41 Ver también Le Fer, París, 4-X-1881, p. 6.

42 El Diluvio, 1-VII-1882, ed. de la mañana, p. 5584

43 La Vanguardia, 16-IX-1883, p. 22, y La Publicidad, 23-IX1883, p. 4.

44 La Publicidad, 21-X-1887, p. 4.

45 La Publicidad, 7-II-1889, p. 4; 7-VII-1889, p. 4, y 5-VIII-1890 p. 2. La noticia de este último se refiere a una huelga de los obreros de la empresa, iniciada el día anterior a consecuencia de una decisión de Anglés, y da el número de huelguistas, treinta y cuatro, sin indicar si se trata de toda la plantilla o solo una parte.

46 La Publicidad, 4-IX-1892, p. 4; 1-I-1893, p. 4, y 1-XI-1897, p. 4 La Vanguardia, 9-XII-1892, p. 2, y 13-VI-1897, p. 2.

47 La Vanguardia, 3-XII-1900, 4

48 El Diluvio, 17-XII-1901, ed. de la mañana, p. 6.

49 Anuncio citado de La Publicidad, 7-II-1889, p. 4.

50 Así figura en la noticia de una huelga de los obreros de la empresa, en La Publicidad, 5-VIII-1890, p. 2, y en un anuncio en el mismo periódico, 8-XI-1891, p. 1.

51 Hace algún tiempo se vendían en Internet dos sobres de la empresa, matasellados en 1902 y 1903, en cuyo membrete figuraba "Directores J. y E. Anglés". Por otra parte, José Anglés fue el solicitante de la aprobación de un contador de CFC, concedida por Real Orden de 10 de julio de 1903 (Gaceta del 18, p. 1544).

52 Véase el Bulletin de l'Association Amicale des Ingénieurs Anciens Élèves de l'École des Ponts et Chaussées de France, juillet 1908, p. 44, y el decreto de concesión del grado de caballero de la Legión de Honor, en Journal Officiel de la République Française, 25-VII-1900, p. 4873

53 El Diluvio, 24-II-1902, ed. de la tarde, 6.

54 Gaceta, 9-X-1904, pp. 122-127.

55 Esta se menciona en la nota 36

56 Como muestra de lo que se decía de los contadores, véase el apartado que les dedicó El Diluvio, 30-XI-1900, pp. 10-11, en el artículo "Todo el estómago de las empresas del gas". Al año siguiente este periódico fue un constante animador de la huelga. Valga, a modo de ejemplo, lo que publicó sobre ella el 2-III-1901, ed. de la mañana, pp. 7-9, cuando acababa de comenzar.

57 Gaceta, 7-VIII-1905, pp. 487-488

58 Gaceta, 25-IX-1905, pp. 1134-1135. La disposición señala que la petición fue elevada al Ministerio por el gobernador civil de Barcelona el 4 de septiembre, y que hacía referencia a una real orden de 3 de agosto (no publicada en la Gaceta), de modo que Ciervo y Triana hubieron de firmarla entre ambas fechas.

59 Real Orden de 21 de febrero de 1907 (Gaceta del 26, p. 738). La modificación de las instrucciones se había publicado en Gaceta, 13-VI-1906, pp. 1021-1023. 
60 Real Orden de 23 de mayo de 1906 (Gaceta del 30, p. 833).

61 Gaceta, 3-X-1901, p. 52. Los catedráticos de la Universidad de Barcelona, Eduard Fontseré i Riba, de mecánica racional, y Manuel Riquelme Sánchez, interino de física general, fueron seguramente los firmantes del preceptivo dictamen favorable, que parece se hizo público (La Publicidad, 9-I-1902, ed. de la mañana, p. 2).

62 AHOEPM, exp. P 28125.

63 Inscripción del nacimiento de Juan, en Arxiu Municipal Contemporani de Barcelona (AMCB), registre civil, naixements, 1857, llibre 3r, no 3003. La de Pedro no se ha localizado.

64 La carrera militar de Cicio, nacido en 1849 y seguramente el mayor de los hermanos, que sirvió muchos años en Filipinas, puede seguirse en los escalafones del Arma de Infantería y el Diario Oficial del Ministerio de la Guerra.

65 Arxiu Històric de Protocols de Barcelona (AHPB), notari Pere Montruit i Serrador, llibre 1435/16, fols. 944-949.

66 El Mundo Científico, Barcelona, 27-XII-1902, pp. 822-826 (ilustrado con figuras).

67 El 5 de diciembre (AOEPM, exp. P 30791). El inventor pidió un certificado de adición a esta patente, el 22 de julio de 1903 (exp. P 32218).

68 Gaceta, 15-I-1903, p. 198

69 Edición de la mañana, p. 6089.

70 El Ingeniero, suplemento al n 499 de Madrid Científico, 1-I1905, p. 102.

71 Anuario del Comercio, de la Industria, de la Magistratura y de la Administración, Madrid, 1906, p. 56 de anuncios.

72 AHPB, notari Pere Montruit i Serrador, llibre 1435/25, fols. 262-263.

73 El Diluvio, 10-XII-1907, ed. de la tarde, pp. 4-5.

74 La Actualidad, Barcelona, 1-VIII-1911, p. 35.

75 Véase, por ejemplo, El Pla de Bages, Manresa, 5-II-1912, p. 3.

76 Véase también El Socialista, Madrid, 3-IV-1913, y Anuario Estadístico de la Ciudad de Barcelona, 1912, pp. 660-661.

77 AHOEPM, exps. P 46555 y P 49766.

7813 de abril de 1910 (Gaceta del 7-V, p. 262) y 14-XII-1911 (Gaceta del 4-I-1912, p. 34)

79 La leyenda completa de la placa es: "Casimiro Abellanet S. en C. / Fabricación de contadores y material para fábricas de gas / Sistema Boix / Con privilegio exclusivo / Barcelona", que rodea a "Contador 3 mecheros / no 10418 / 420 litros por hora / 1915".

80 Calle Marqués del Duero (actual Avinguda del Paral·lel), 122 (Arquitectura y Construcción, 1918, en "Libro segundo / secciones técnica e indicadora clasificadas por provincias", p. 62). Abellanet solicitó permiso municipal para instalar un motor eléctrico en esa dirección (La Publicidad, 29-V-1915, p. 3)

81 El Día, Manresa, 30-IX-1933, p. 1.

82 Artículo 109 del decreto ya citado de 7 de octubre de 1904.

83 AHPB, notari Guillem August Tell i Lafont, llibres 1428/66, fols. 3173-3184, y 1428/67, fols. 569-573.

84 Solicitud de 4 de agosto de 1908 de patente de introducción de "un contador con rueda de paletas". (AHOEPM, exp. P 43841). Este archivo conserva también las solicitudes de las marcas de contadores de agua "Sma. FRAGER" ( $n$ 14888, 10 de abril) y "FRAGER" (no 15258, 29 de septiembre).

85 El Liberal, Madrid, 10-XI-1907, 2. También en El Heraldo, mismos día y página.

86 Fotografías en Canet, Emilio, dir. (1912) Barcelona artística e industrial. Barcelona.

87 La Ilustración Artística, Barcelona, 6-II-1911, 98, y Nuevo Mundo, Madrid, 9-II-1911 (ambas con fotografías).
88 Véase, por ejemplo, la primera crónica encontrada en La Publicidad, 11-IV-1913, p. 2, y la última, que no hace referencia al Congreso, en Revista llustrada de Banca, Ferrocarriles, Industria y Seguros, 25-IX-1913, p. 491.

89 Gaceta, 26-VII-1912, pp. 214-215; 14-II-1917, p. 387, y 4-VI1917 , p. 618.

90 Revue industrielle, París, 15-VII-1905, pp. 275-276.

91 Gaceta de Madrid, 26-VII-1912, pp. 214-215; 14-II-1917, p. 387, y 4-VI-1917, p. 618.

92 AHPB, notari Antoni Par i Tusquets, llibre 1442/117, fols. 2475-2484.

93 AHPB, notari Antoni Par i Tusquets, llibre 1442/117, fols. 2481-2484. Viteau pertenecía a la promoción de 1899 de l'École Nationale Supérieure des Mines, en cuyo Bulletin de la Association amicale des élèves pueden seguirse los pasos de su actividad profesional, primero en Francia y después en Argentina, así como que últimamente había combatido en la Gran Guerra, resultando herido. Camacho había nacido en París en 1882 , hijo de padre mejicano y madre francesa, nacionalidad a la que renunció en 1903 (Bulletin des lois de la République Française, décrets d'intérêt local ou particulier, deuxième semestre de 1903, p. 137).

94 Producción, Madrid, 5-XII-1920, s/p.

95 AHPB, notari Antoni Par i Tusquets, llibre 1442/148, nº 734 a 736, fols. 2606-2617.

96 El Financiero, Madrid, 23-IX-1921, p. 2110.

97 El Auxiliar de la Ingeniería y Arquitectura, Madrid, 25-XII-1926, s/p.

98 Reales Órdenes de 4 de septiembre de 1925 y 24 de abril de 1929 (Gaceta, 13-X, p. 192, y 23-V, p. 1113, respectivamente). Patente solicitada el 1 de octubre de 1920 (AHOEPM, exp. P 75860).

$99 N^{\text {os }} 650$ y 2486 de 1862 , y 1548 de 1865 . Ver "Patents for Inventions. Abridgements of Specifications Relating to the Production and Applications of Gas. (Excepting Gas Engines.) Part II.-A. D. 1859-1866 [...] London [...] 1871".

100 Véanse, entre otras fuentes, "Centenary link with Europe", Gas Journal, 4-VIII-1965, p. 134, y "Kromschröder through the ages", Profiles, the Elster customer magazine, 1/2015, pp. 17-19.

101 Gaceta, 12-VIII-1908, pp. 632-633. Seguramente fue el objeto de la patente de introducción y certificado de adición solicitados el 12 de enero de 1909 y 26 de septiembre de 1911 (AOEPM, exps. P 44741 y 51387 ).

102 Gaceta, 14-IV-1910, pp. 105-106, y 5-IX-1912, p. 542.

103 Contadores en Gaceta, 4-VII-1922, p. 47; 13-X-1927, p. 259; 28-VI-1930, p. 1972; 16-VII-1931, pp. 471-472, y 3-X-1932, p. 43. Aparatos de pago previo en Gaceta, 28-VI-1930, p. 1971, y 10-X-1932, p. 191. En Gaceta, 14-III-1929, p. 1930, aprobación y descripción de una "válvula automática aplicable a los contadores de gas secos".

104 La Vanguardia, 28-I-1922, p. 23.

105 Registro Mercantil de Barcelona (RMB), folios 50-52, tomo 271 , hoja 18052 , inscripciones 1 a y 2 a.

$106 \mathrm{RMB}$, folios 52-53, tomo 271, hoja 18052, inscripción 3a.

107 La Veu de Catalunya, 31-V-1936, ed. matí, p. 19.

108 Gaceta, 26-VIII-1912, 453-454.

109 En Inglaterra patente $\mathrm{n}$ - 2090 de ese año, y en Estados Unidos no 217,093 .

110 Gaceta, 23-VIII-1931, pp. 1423-1424; 22-IX-1932, p. 2118; 7-XII-1932, pp. 1708-1709, у 25-I-1933, p. 597 (aparato pago previo).

111 Gaceta, 7-XI-1933, p. 908.

112 Gaceta, 20-V-1934, anexo único, pp. 562-563.

113 La Gaceta Industrial, 10-II-1881, p. 364 


\section{BIBLIOGRAFÍA}

Alayo, Joan Carles; Barca, Francesc-X. (2011), La tecnología del gas a través de su historia, Fundación Gas Natural Fenosa.

Arroyo, Mercedes (2002), "El gas de Madrid y las compañías de crédito extranjeras en España (1856-1890)", Scripta Nova, 6 (131), [en línea], disponible en: http:// www.ub.edu/geocrit/sn/sn-131.htm [consultado el 26/04/2021]

Fábregas, Pere-A. (2020), "Los primeros clientes del gas del alumbrado en España: Barcelona 1843-1847. Estructuras, categorías y patrones". En: Bartolomé, Isabel; Fernández-Paradas, Mercedes; Araujo, Jesús Miras (eds.), Cercanas pero distintas: la desigual trayectoria de la industria del gas en las regiones del sur de Europa (siglos $X(X-X X)$, Madrid, Marcial Pons, pp. 121-146.
Fernández-Paradas, Mercedes (2016), “La regulación del suministro de gas en España (1841-1936)", Revista de Historia Industrial, 25 (61), monográfico 1.

Giguel, Paulette (2014), La Compagnie des Compteurs, Presses Universitaires de Rennes.

Pelpel, Jacques (1987), "Historique de la Compagnie des compteurs", Culture Technique, sin. vol. (17), pp. 20-23.

Sánchez Miñana, Jesús; Sánchez Ruiz, Carlos (2020), “Ciervo en la Barceloneta (1861-circa 1960): Ascenso y declive de una empresa auxiliar de la industria del gas, primera fábrica de contadores en España". En: Bartolomé, Isabel; Fernández-Paradas, Mercedes; Araujo, Jesús Miras (eds.), Cercanas pero distintas: la desigual trayectoria de la industria del gas en las regiones del sur de Europa (siglos XIX-XX), Madrid, Marcial Pons, pp. 165-182.

Sastre, Miguel (1915), Las huelgas en Barcelona, Barcelona, Imprenta Editorial Barcelonesa, S.A. 